\title{
One-pot solvent exchange preparation of non-swellable, thermoplastic, stretchable and adhesive supramolecular hydrogels based on dual synergistic physical crosslinking
}

\author{
Qian Feng ${ }^{1}$, Kongchang Wei ${ }^{1,2}$, Kunyu Zhang ${ }^{1}$, Boguang Yang ${ }^{1,2}$, Feng Tian ${ }^{3}$, Guixue Wang ${ }^{4}$ and \\ Liming Bian ${ }^{1,2,5,6,7}$
}

With their unique properties of self-healing and viscoelasticity, physically crosslinked supramolecular hydrogels are promising materials for soft robotics, wearable electronics and biomedical applications. However, the weak mechanical properties of supramolecular hydrogels, especially those prepared with natural polymers, limit their wide-spread application, and swelling is one of the key factors that contributes to the weakening of hydrogels. Herein, we utilize a simple one-pot solvent exchange method to prepare non-swellable, thermoplastic and tough supramolecular gelatin hydrogels based on two synergistic physical crosslinkings, namely, the self-assembled tri-helix structure of gelatin and the hydrophobic aggregation of gelatin-grafted and free hydrophobic motifs. The obtained hydrogels possess a stable water content above $70 \%$ with extended incubation in water. These hydrogels are highly malleable upon heating but are extremely stretchable and tough after cooling to room temperature. Furthermore, the supramolecular gelatin hydrogels exhibit robust adhesion to various material surfaces and minimal cytotoxicity. NPG Asia Materials (2018) 10, e455; doi:10.1038/am.2017.208; published online 5 January 2018

\section{INTRODUCTION}

Hydrogels, due to their high water content and tunable physical and biological properties, are extensively used in soft robotics, wearable electronics and biomedical applications. ${ }^{1-6}$ Supramolecular hydrogels, which are solely stabilized by physical crosslinkings, such as host-guest interactions, electrostatic attraction and hydrophobic aggregation, have recently received increasing attention due to their unique properties, including self-healing, energy dissipation and viscoelasticity. ${ }^{7-13}$ However, the poor mechanical performance of supramolecular hydrogels, especially hydrogels prepared with natural polymers, which possess superior biocompatibility and bioactivity, remain a major hurdle to the wide-spread application of these hydrogels. To improve the mechanical properties of chemically crosslinked hydrogels, researchers have developed ingenious strategies, including nanocomposite hydrogels, double-network hydrogels, hybrid crosslinking hydrogels and tetra-polyethylene glycol hydrogels. ${ }^{14-22}$ Recently, increasing research attention has been dedicated to enhancing the mechanical performance of physically crosslinked supramolecular hydrogels. One recent study demonstrated very tough physical hydrogels composed of polyampholytes. ${ }^{23}$ Another study showed that pre-assembled host and guest monomers via host-guest complexation effectively strengthened the obtained supramolecular hydrogels. ${ }^{24}$ Dai et al. ${ }^{25}$ recently demonstrated an elegant approach to prepare mechanically strong and highly stable supramolecular hydrogels based on hydrogen bonding. It should be noted that the network structure of supramolecular hydrogels reported by these earlier studies predominantly consists of synthetic polymers, and methods to enhance the mechanical performance of natural polymer-based supramolecular hydrogels remain limited. Our earlier studies showed that pre-organization of host monomers along natural polymers via host-guest interactions before polymerization significantly improved the mechanical robustness of natural polymer-based supramolecular hydrogels, but the toughness of these hydrogels remained low. ${ }^{26,27}$ To the best of our knowledge, there has been no prior report on supramolecular hydrogels prepared with pure natural polymers that exhibit outstanding mechanical toughness.

The poor mechanical properties, including the toughness, of hydrogels can be partially attributed to swelling, which leads to a significant decrease in the crosslinking density in the hydrogels. ${ }^{28-30}$ Compared to the as-prepared hydrogels, a drastic reduction is often observed in the mechanical properties of the swollen hydrogels.

${ }^{1}$ Department of Biomedical Engineering, The Chinese University of Hong Kong, Shatin, Hong Kong; ${ }^{2}$ Shun Hing Institute of Advanced Engineering, The Chinese University of Hong Kong, Shatin, Hong Kong; ${ }^{3}$ Shanghai Institute of Applied Physics, Chinese Academy of Sciences, Shanghai, China; ${ }^{4}$ State and Local Joint Engineering Laboratory for Vascular Implants, Key Laboratory for Biorheological Science and Technology of Ministry of Education, College of Bioengineering, Chongqing University, Chongqing, People's Republic of China; ${ }^{5}$ Shenzhen Research Institute, The Chinese University of Hong Kong, Shenzhen, China; ${ }^{6}$ China Orthopedic Regenerative Medicine Group (CORMed), Hangzhou, China and ${ }^{7}$ Centre of Novel Biomaterials, The Chinese University of Hong Kong, Hong Kong

Correspondence: Professor L Bian, Department of Biomedical Engineering, The Chinese University of Hong Kong, ShaTin 999077, Hong Kong.

E-mail: Ibian@cuhk.edu.hk

Received 23 June 2017; revised 14 September 2017; accepted 1 October 2017 
Solvent exchange from organic to aqueous solvent induces hydrophobic aggregation of amphiphilic polymers. ${ }^{31,32}$ Previous studies have shown the potential of this method to prepare non-swellable hydrogels by carefully adjusting the mass ratio between hydrophilic and hydrophobic blocks of the amphiphilic polymers. ${ }^{33}$ However, these self-assembled hydrogels stabilized by hydrophobic aggregations are generally weak and have poor elasticity, thereby requiring additional crosslinking to further reinforce the network structure. Natural polymers, such as collagen and its derivative product, gelatin, can self-assemble into stable and rigid tertiary structures, such as trihelices, at certain temperatures. ${ }^{34,35}$ These tertiary structures have an indispensable role in stabilizing the extracellular matrices of tissues and organs and are ideal crosslinkers for strengthening supramolecular hydrogels prepared by solvent exchange. ${ }^{36}$

Herein, we propose a facile one-pot solvent exchange approach to prepare extremely stretchable, tough and non-swellable supramolecular gelatin hydrogels based on two physical crosslinking mechanisms, self-assembly of the tri-helix structure of gelatin and aggregation of free and gelatin-grafted hydrophobic groups. Exhibiting classic thermoplastic behaviors, the obtained supramolecular gelatin hydrogels become highly malleable upon heating but are extremely stretchable and tough after cooling to room temperature. Furthermore, the supramolecular gelatin hydrogels exhibit robust adhesion to various material surfaces and minimal cytotoxicity.

\section{MATERIALS AND METHODS}

\section{Materials}

Gelatin (type A), 1-adamantyl isothiocyanate, phenyl isothiocyanate, dimethylsulfoxide-d6 (DMSO-d6), ethidium bromide and dimethylmalonic acid were purchased from Sigma, Hong Kong, China. DMSO and acetone were purchased from Fisher Scientific, Hong Kong, China. Phosphate-buffered saline, $\alpha$-minimum essential medium, penicillin/streptomycin, L-glutamine, calcein AM, fetal bovine serum and Trizol were obtained from Gibco, Hong Kong, China. Human mesenchymal stem cells (hMSCs) were obtained from Lonza, Benicia, CA, USA.

\section{Methods}

Hydrogel preparation. Gel-Ada hydrogel: First, $5 \mathrm{~g}$ of gelatin (type A) was dissolved in $50 \mathrm{ml}$ of DMSO containing 1-adamantyl isothiocyanate $(1.125,1.5$ or $3.0 \mathrm{~g}$ ) at $60^{\circ} \mathrm{C}$. After stirring for $5 \mathrm{~h}$ at $60^{\circ} \mathrm{C}$, the resulting mixture was dialyzed against Deionized water (DI water) for 3 days at room temperature (6 kDa cutoff dialysis membrane). The obtained Gel-Ada hydrogel was immersed in DI water and stored at $-20^{\circ} \mathrm{C}$ in a freezer. Unless otherwise mentioned, Gel-Ada hydrogels prepared with $1.5 \mathrm{~g}$ of 1-adamantyl isothiocyanate were used for the following measurements.

Gel-Phe hydrogel: First, $5 \mathrm{~g}$ of gelatin (type A) was dissolved in $50 \mathrm{ml}$ of DMSO at $60^{\circ} \mathrm{C}$. Phenyl isothiocyanate $(0.6975,0.93$ or $1.86 \mathrm{ml})$ was added to the $10 \%$ gelatin solution, which was then stirred for $5 \mathrm{~h}$ at $60^{\circ} \mathrm{C}$. The resulting mixture was dialyzed against DI water for 3 days at room temperature $(6 \mathrm{kDa}$ cutoff dialysis membrane). The obtained Gel-Phe hydrogel was immersed in DI water and stored at $-20^{\circ} \mathrm{C}$ in a freezer. Unless otherwise mentioned, Gel-Phe hydrogels prepared with $0.93 \mathrm{ml}$ of phenyl isothiocyanate were used for the following measurements.

Small-angle X-ray scattering test. Small-angle X-ray scattering (SAXS) experiments were performed on the BL16B beam line of the Shanghai Synchrotron Radiation Facility. The sample-to-detector distance was $2000 \mathrm{~mm}$, and the $\mathrm{X}$-ray wavelength was $\lambda=0.124 \mathrm{~nm}$. Two-dimensional SAXS data were converted into one-dimensional intensity $I(q)$ as a function of scattering vector $q$ $[q=(4 \pi / \lambda) \sin \theta]$ by circular averaging, where $2 \theta$ is the scattering angle. A hydrogel specimen with a disk shape $(d=5 \mathrm{~mm}, h=1 \mathrm{~mm})$ was used for SAXS analysis.
Proton nuclear magnetic resonance analysis. Gel-Ada hydrogel: To prepare the sample, $0.1 \mathrm{~g}$ of prepared Gel-Ada hydrogel was redissolved in $10 \mathrm{ml}$ DMSO at $60^{\circ} \mathrm{C}$. The solution was dialyzed against DMSO for 3 days to remove unreacted 1-adamantyl isothiocyanate molecules and then dialyzed against DI water for 3 days. Next, the liquid was lyophilized for 3 days at $-104^{\circ} \mathrm{C}$ to obtain pure 1-adamantyl isothiocyanate-modified gelatin polymer. To calculate the substitution degree of the adamantyl groups in the gelatin, $10 \mathrm{mg}$ of polymer was dissolved in $500 \mu \mathrm{l}$ DMSO-d6 with dimethylmalonic acid as an internal reference for proton nuclear magnetic resonance $\left({ }^{1} \mathrm{H}\right.$ NMR) analysis at $25^{\circ}$ C. Another $0.1 \mathrm{~g}$ of Gel-Ada hydrogel was lyophilized for 3 days directly. Next, to calculate the total amount of adamantyl groups in the Gel-Ada hydrogel, $10 \mathrm{mg}$ of dried hydrogel was dissolved in $500 \mu \mathrm{l}$ DMSO-d6 with dimethylmalonic acid as an internal reference for ${ }^{1} \mathrm{H}$ NMR analysis at $25^{\circ} \mathrm{C}$.

Gel-Phe hydrogel: To prepare the sample, $0.1 \mathrm{~g}$ of prepared Gel-Phe hydrogel was redissolved in DMSO to form a solution with $1(\mathrm{w} / \mathrm{v}) \%$ concentration. The resulting solution was dialyzed against DMSO for 3 days and then DI water for 3 days. Pure phenyl isothiocyanate-modified gelatin polymer was obtained after 3 days of freeze drying. The substitution degree of phenyl groups in the gelatin was determined by ${ }^{1} \mathrm{H}$ NMR analysis (Bruker Advance $400 \mathrm{MHz}$ spectrometer, Beijing, China) using the same method as that used for the Gel-Ada hydrogel. At the same time, $0.1 \mathrm{~g}$ of Gel-Phe hydrogel was also freeze-dried directly, and then, $10 \mathrm{mg}$ of dried hydrogel was dissolved in $500 \mu \mathrm{l}$ DMSO-d6 with dimethylmalonic acid as an internal reference for ${ }^{1} \mathrm{H}$ NMR analysis at $25^{\circ} \mathrm{C}$. For both the Gel-Ada hydrogel and Gel-Phe hydrogel, pure gelatin was used as the control group.

Tensile mechanical measurement. The width and thickness of the specimen were 20 and $6 \mathrm{~mm}$, respectively. The specimen was clamped by two aluminum alloy holders to produce a $3 \mathrm{~mm}$ gauge length. The tension tests were performed at an extension speed of $1 \mathrm{~mm} \mathrm{~s}^{-1}$. The broken samples can be reshaped in a glass mold, which is used to remold the sample for the tension test at temperatures above $30^{\circ} \mathrm{C}$. For the notch tensile test, the samples were cut in the middle by $5 \mathrm{~mm}$ ( $20 \%$ of the total width). The tensile fatigue test was performed with a tensile strain of $\lambda 10$ for 10 cycles ( $60 \mathrm{~s}$ every cycle). For the recovery test, after the above mentioned continuous 10 cycles test $\left(1 \mathrm{~mm} \mathrm{~s}^{-1}\right)$, the hydrogels were rested for $5 \mathrm{~min}$ before the eleventh cycle test. For the tensile relaxation test, the hydrogel sample was stretched to $\lambda 10$ at a speed of $1 \mathrm{~mm} \mathrm{~s}^{-1}$, and then, the strain was maintained, and the change in stress was recorded.

Rheological experiment. Rheological tests were performed using a Malvern KINEXUS rheometer (Shanghai, China) using $20 \mathrm{~mm}$ diameter plates (plate to plate) with a $1 \mathrm{~mm}$ gap. The hydrogels were homogeneously distributed between the top and bottom plates of the rheometer. The time sweep was recorded at a strain of $1 \%$ and a frequency of $10 \mathrm{~Hz}$. During the time sweep, the temperature of the samples was increased from 25 to $45^{\circ} \mathrm{C}$ and then decreased from 45 to $25^{\circ} \mathrm{C}$ (repeated for five cycles) to examine the effect of the temperature on the modulus of the Gel-Ada and Gel-Phe hydrogels. For the frequency sweep test, the strain was $1 \%$, and the frequency was changed from 0.1 to $10 \mathrm{~Hz}$.

Lap-shear test. Aluminum alloy: To prepare the samples, $0.5 \mathrm{ml}$ of Gel-Ada or Gel-Phe hydrogel was spread over a $1.5 \mathrm{~cm} \times 2 \mathrm{~cm}$ area on one aluminum alloy strip (total size: $7 \mathrm{~cm}$ length $\times 2 \mathrm{~cm}$ width), and then, the other strip was brought into contact with the hydrogel-covered strip in a $45{ }^{\circ} \mathrm{C}$ water bath for $10 \mathrm{~min}$. Next, the samples were cooled to room temperature before the lapshear test. The maximum adhesive strength was determined by completely separating the overlapped aluminum alloy plates at a cross-head speed of $1 \mathrm{~mm} \mathrm{~s}^{-1}$. Each sample was measured four times.

Glass: To prepare the samples, $0.5 \mathrm{ml}$ of Gel-Ada or Gel-Phe hydrogel was spread over a $1.5 \mathrm{~cm} \times 2 \mathrm{~cm}$ area on one glass strip (total size: $7 \mathrm{~cm}$ length $\times 2 \mathrm{~cm}$ width), and then, the other strip was brought into contact with the hydrogel-covered strip in a $45^{\circ} \mathrm{C}$ water bath for $10 \mathrm{~min}$. Next, the samples were cooled to room temperature before the lap-shear test. The maximum adhesive strength was determined by completely separating the overlapped glass plates at a cross-head speed of $1 \mathrm{~mm} \mathrm{~s}^{-1}$. Each sample was measured four times. 
Porcine skin: Fresh porcine skin was cut into a rectangular section $4 \mathrm{~cm} \times 1.5 \mathrm{~cm}$ in size and $\sim 3 \mathrm{~mm}$ thick and wetted by soaking in water before use. Next, $0.5 \mathrm{ml}$ Gel-Ada or Gel-Phe hydrogel was spread over a $1.5 \mathrm{~cm} \times 2 \mathrm{~cm}$ area on one strip, and then, the other strip was brought into contact with the hydrogel-covered strip in a $45^{\circ} \mathrm{C}$ water bath for $10 \mathrm{~min}$. Next, the samples were cooled to room temperature before the lap-shear test. The maximum adhesive strength was determined by completely separating the overlapped skins at a cross-head speed of $1 \mathrm{~mm} \mathrm{~s}^{-1}$. Each sample was measured four times.

In vitro biocompatibility test. Briefly, 50000 hMSCs were seeded into a confocal dish, and then, $0.5 \mathrm{ml}$ of the hydrogel was added to the confocal dish to co-culture with the hMSCs. At day 5 and day 10, the cell samples were collected and stained with calcein AM (live) and ethidium bromide (dead) to test the cell viability.

\section{RESULTS AND DISCUSSION}

The dual physical crosslinking of supramolecular gelatin hydrogels Gelatin, a hydrolysis product of natural collagen, is widely used in various biomedical applications due to its good biocompatibility, intrinsic bioactivity, abundance and relatively low antigenicity compared with collagen..$^{37,38}$ Gelatin solutions exhibit a thermo-reversible Sol-Gel transition and form gels upon cooling below $30^{\circ} \mathrm{C}$. The SolGel phase transition of gelatin solutions is attributed to the supramolecular assembly and disassembly of the tri-helix structure as a result of the formation and breakage of intermolecular hydrogen bonds at low and high temperatures, respectively. However, the rigidity of the gelatin tri-helix renders pure gelatin hydrogels very brittle. In this study, we incorporated a second form of supramolecular interaction, hydrophobic interaction, to enhance the toughness of the supramolecular gelatin hydrogels.

As illustrated in Figure 1, all reagents, including gelatin and hydrophobic small molecules (phenyl isothiocyanate or 1-adamantyl isothiocyanate) were dissolved and reacted in DMSO at $60^{\circ} \mathrm{C}$ for $5 \mathrm{~h}$, thereby grafting the hydrophobic groups to the gelatin backbone via isothiocyanate-amino coupling. The molar ratio between the hydrophobic molecules and the gelatin amino groups was set at a value significantly larger than 1 to ensure the presence of excess unreacted hydrophobic molecules in the final reaction mixture (Supplementary Figures S4 and S6), which was subsequently dialyzed directly against DI water at room temperature. During dialysis, cooling prompts the gelatin molecules to form a tri-helix structure, while the gradual solvent exchange from DMSO to DI water induces aggregation of the unreacted and grafted hydrophobic molecules. Driven by these two spontaneous physical interactions, bulk hydrogels (Gel-Phe: phenylisothiocyanate used as the hydrophobic molecule; Gel-Ada: 1 -adamantyl isothiocyanate used as the hydrophobic molecule) are gradually formed inside the dialysis bag after 3 days of dialysis. It should be noted that the presence of an excess amount of unreacted free hydrophobic small molecules is essential to the preparation of such supramolecular hydrogels. Dialysis of the final reaction mixture in DMSO first before transferring to DI water resulted in no hydrogel formation. This indicates that in the absence of free hydrophobic small molecules (removed by first dialyzing against DMSO), the gelatin polymers with grafted hydrophobic molecules alone are not capable of forming hydrogels under solvent exchange conditions.

We attempted to use different amounts of hydrophobic molecules to prepare hydrogels (1-adamantanyl isothiocyanate: $1.125,1.5$ or 3.0 g+gelatin: $5 \mathrm{~g}$; phenyl isothiocyanate: $0.6975,0.93$ or $1.86 \mathrm{ml}$ +gelatin: $5 \mathrm{~g}$ ). The results show that the addition of low amount of hydrophobic molecules (1.125 g 1-adamantanyl isothiocyanate and $0.6975 \mathrm{ml}$ phenyl isothiocyanate) only leads to the formation of precipitates instead of hydrogels. The estimated minimum amount of hydrophobic small molecules required to prepare the Gel-Ada and Gel-Phe hydrogels is $0.3 \mathrm{~g} 1$-adamantynal isothiocyanate per gram of gelatin and $0.186 \mathrm{ml}$ phenyl isothiocyanate per gram of gelatin, respectively. Furthermore, ${ }^{1} \mathrm{H}$ NMR analysis of the freeze-dried GelAda and Gel-Phe hydrogels (prepared with the minimum amount of hydrophobic small molecules, that is, $1.5 \mathrm{~g}$ of 1-adamantanyl isothiocyanate or $0.93 \mathrm{ml}$ of phenyl isothiocyanate; Supplementary Figures S5 and S7) show that each gram of dried Gel-Ada hydrogel contains $\sim 0.13 \mathrm{~g}$ of free 1-adamantyl isothiocyanate and $0.87 \mathrm{~g}$ of adamantine-grafted gelatin, and each gram of dried Gel-Phe hydrogel contains $\sim 0.08 \mathrm{~g}$ of free phenyl isothiocyanate and $0.92 \mathrm{~g}$ of phenylgrafted gelatin. When the amount of hydrophobic small molecules used for hydrogel preparation increases, the content of free nonconjugated hydrophobic small molecules in the freeze-dried Gel-Ada and Gel-Phe hydrogels slightly increases (Supplementary Figures S5 and S7). However, this varying content of hydrophobic small molecules does not significantly influence the mechanical properties of the Gel-Ada and Gel-Phe hydrogels (Supplementary Figure S9). Our collective findings show that while there is a minimum amount of hydrophobic small molecules required for hydrogel formation, further increasing the amount of hydrophobic molecules over this minimum threshold does not significantly alter the mechanical properties of the obtained supramolecular hydrogels. Therefore, unless otherwise stated, the Gel-Ada hydrogels prepared with $1.5 \mathrm{~g}$ of 1-adamantyl isothiocyanate and Gel-Phe hydrogels prepared with $0.93 \mathrm{ml}$ of phenyl isothiocyanate were used for the following measurements.

SAXS analysis revealed a broad correlation peak spanning a wide range of scattering vectors $(q(4 \pi / \lambda) \sin \theta)$ from 0.5 to $1.5 \mathrm{~nm}^{-1}$ for the Gel-Ada hydrogels, whereas no peaks were observed for pure gelatin hydrogel at $20^{\circ} \mathrm{C}$ (Supplementary Figure S1a). Such a broad peak may be attributed to the random distribution of hydrophobic aggregations rather than the gelatin tri-helix structure in the Gel-Ada hydrogel matrix. Furthermore, the SAXS peak of the Gel-Ada hydrogels shifted slightly to the left upon increasing the temperature to $45^{\circ} \mathrm{C}$ (Supplementary Figure S1b). Using Bragg's law (Equation (1)):

$$
d=\frac{\lambda}{2 \sin \theta}=\frac{2 \pi}{q_{\max }}
$$

where $d$ is the spacing between the scattering centers and $q_{\max }$ is the peak position, we can estimate the spacing between the hydrophobic aggregation as $8.92 \mathrm{~nm}$ at $20^{\circ} \mathrm{C}$ and $10.67 \mathrm{~nm}$ at $45^{\circ} \mathrm{C}$. We speculate that the network structure of supramolecular Gel-Ada hydrogels is stabilized by the synergistic action of the homogeneously mixed gelatin tri-helix structures and hydrophobic aggregations. Increasing temperature leads to the disassembly of the gelatin tri-helix, thereby leading to increased spacing among the hydrophobic aggregations. We also attempted to use hyaluronic acid and agarose to fabricate selfassembled supramolecular hydrogels using the same solvent exchange approach. Because of the absence of amino groups in both hyaluronic acid and agarose, we grafted 1-adamantane acetic acid (excess amount) as hydrophobic moieties to hyaluronic acid (HA-Ada) or agarose (Aga-Ada) by reaction with the hydroxyl groups of these two polymers. However, dialysis of the reaction DMSO solutions of HAAda and Aga-Ada in DI water at room temperature only yielded powdered precipitates and brittle hydrogel fragments, respectively (Supplementary Figure S2). We speculate that the powdered precipitates are due to the aggregation of unreacted hydrophobic molecules because HA-Ada remains water-soluble under room temperature. Although agarose can self-assemble to a coil-helix structure at room temperature, the semi-rigid agarose backbone may hinder the homogeneous and synergistic precipitation between the 


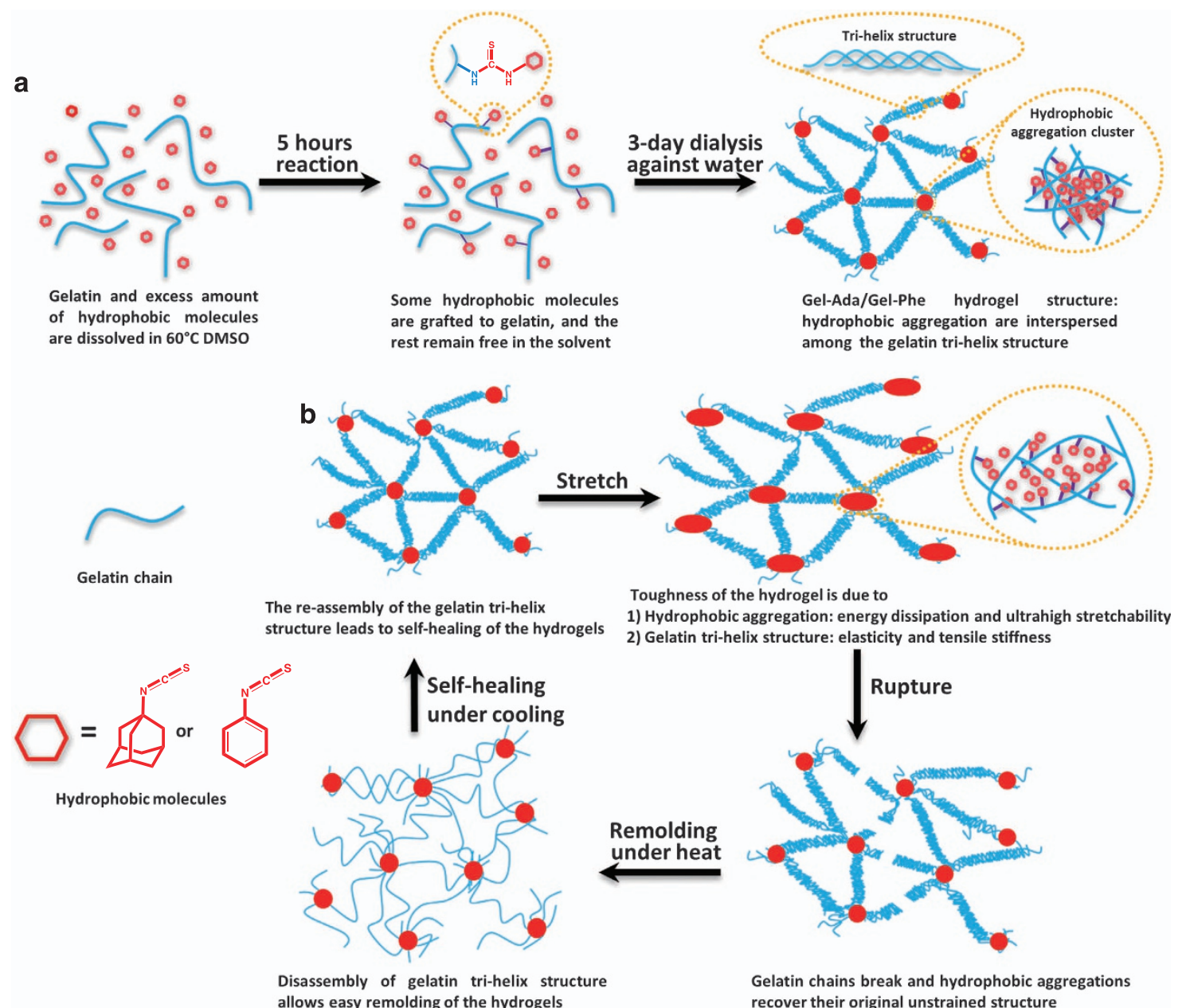

Figure 1 (a) Preparation of Gel-Ada/Gel-Phe supramolecular gelatin hydrogels with dual physical crosslinking mechanisms, that is, self-assembly of gelatin trihelix and aggregation of free and grafted hydrophobic motifs. (b) The unique dual physical crosslinking of the hydrogel network structure produces the highly stretchable and thermoplastic properties of the Gel-Ada/Gel-Phe hydrogels. The deformation of reversible hydrophobic aggregation leads to the energy dissipation and ultrahigh stretchability of the supramolecular hydrogels. The rigidity of the gelatin tri-helix structure contributes to the elasticity and tensile stiffness. The synergistic reaction of these two crosslinking mechanisms leads to the substantial toughness of the hydrogel. After rupture in the tensile test, the hydrogel samples can be remolded by heating and self-healed by cooling.

agarose coil-helix and hydrophobic small molecules. These findings suggest that the formation of bulk Gel-Ada and Gel-Phe hydrogels is driven by the coordinated self-assembly of gelatin and the precipitation of aggregated hydrophobic small molecules (both grafted and nongrafted) in a synergistic manner.

\section{The thermoplasticity of supramolecular gelatin hydrogels}

Both the Gel-Phe and Gel-Ada hydrogels show thermoplastic properties. These two hydrogels are highly malleable at high temperature $\left(45^{\circ} \mathrm{C}\right.$ ) but become rigid and elastic at low temperature (room temperature, $25^{\circ} \mathrm{C}$ ). For example, after a brief incubation in $45^{\circ} \mathrm{C}$ DI water for 1-2 min, a piece of Gel-Phe hydrogel (yellow) and GelAda hydrogel (white) can be hand-drawn into elongated cords (Figure 2a). After cooling to room temperature, the two hydrogel cords maintain their morphology and are robust enough to be knotted into a 'bow' structure. The knotted hydrogel cords can be untangled at low temperature and subsequently remolded to their original shape at high temperature (Supplementary Video 1; Figure 2a). By switching the temperature as described above, we can recycle the used Gel-Phe and Gel-Ada hydrogels and repeatedly alter their morphology, like play dough. The results from our time-sweep rheological test provide further insight into the thermoplastic properties of Gel-Phe and GelAda hydrogels (Figure 2b; Supplementary Figure S8). At low temperature $\left(25^{\circ} \mathrm{C}\right)$, the storage modulus of the Gel-Phe hydrogels $\left(G^{\prime}\right.$, $67 \mathrm{kPa})$ is significantly higher than the loss modulus $\left(G^{\prime \prime}, 7 \mathrm{kPa}\right.$; Figure $2 \mathrm{~b}$ ), indicating that elasticity is dominant in the hydrogels at low temperature. With increasing temperature, both $G^{\prime}$ and $G^{\prime \prime}$ of GelPhe hydrogels decrease, and $\mathrm{G}^{\prime}$ decreases faster than $G^{\prime \prime}$. Therefore, when the temperature reaches $45^{\circ} \mathrm{C}$, the value of $G^{\prime \prime}(2.68 \mathrm{kPa})$ becomes very close to that of $G^{\prime}(1.98 \mathrm{kPa}$; Figure $2 \mathrm{~b})$, and the GelPhe hydrogels show significant viscoelasticity, thereby resulting in the excellent remoldability of the Gel-Phe hydrogels. Subsequent cooling of the Gel-Phe hydrogels to $25^{\circ} \mathrm{C}$ leads to the complete recovery of $G^{\prime}$ and $G^{\prime \prime}$ to the original level (Figure 2b). Such a cyclic temperaturedependent change in the storage modulus and loss modulus can be repeated many times, but only two consecutive cycles are presented for brevity (Figure $2 \mathrm{~b}$ ). The Gel-Ada hydrogels also exhibit reversible and thermoplastic behavior similar to that of the Gel-Phe hydrogels (Supplementary Figure S7). Furthermore, the average values of the storage modulus and loss modulus of the Gel-Ada and Gel-Phe hydrogels measured at $25^{\circ} \mathrm{C}$ in each of the five consecutive cycles of temperature change only increase slightly with increasing cycle 
a
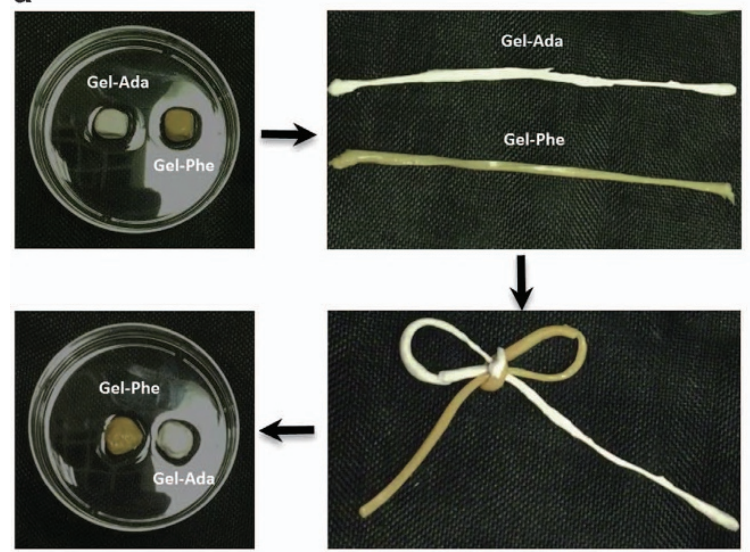

C

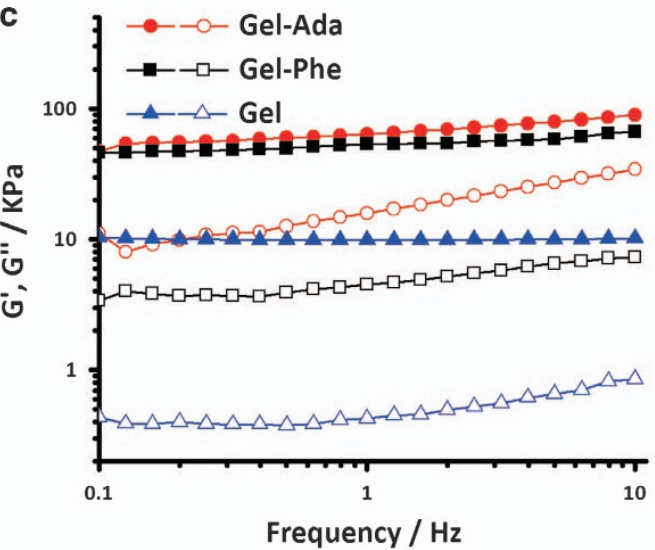

b

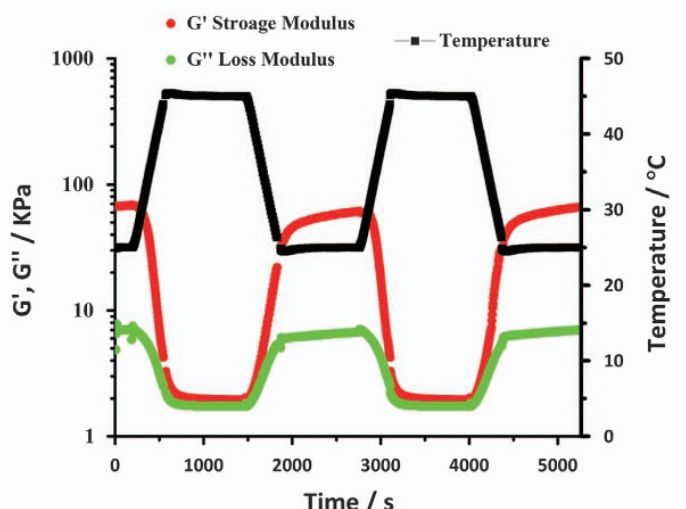

d

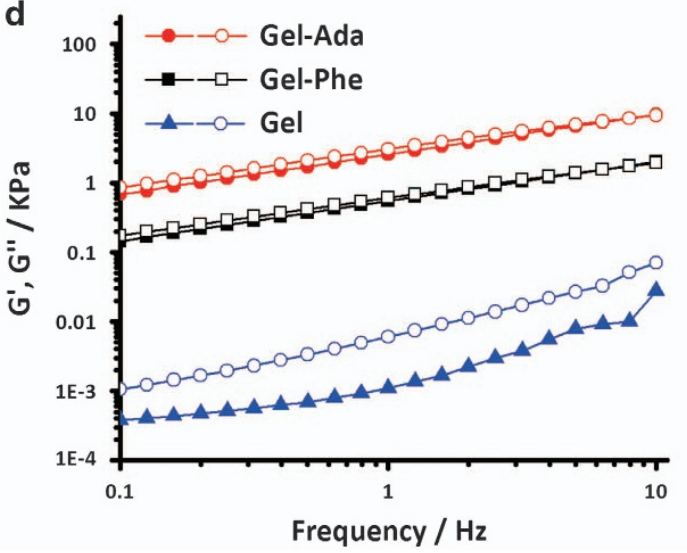

Figure 2 (a) Thermoplastic properties: the Gel-Ada and Gel-Phe hydrogels are highly malleable at high temperature (45 ${ }^{\circ} \mathrm{C}$ ) but become rigid and elastic at low temperature (room temperature, $25^{\circ} \mathrm{C}$ ). For example, after a brief incubation in $45^{\circ} \mathrm{C}$ DI water for $1-2$ min, a piece of the Gel-Phe hydrogel (yellow) and Gel-Ada hydrogel (white) can be hand-drawn into elongated cords. After cooling to room temperature, the two hydrogel cords maintain their morphology and are sufficiently robust to be knotted into a 'bow' structure. The knotted hydrogel cords can be untangled at low temperature and subsequently remolded to their original shape at high temperature. (b) Time sweep rheological analysis of the Gel-Phe hydrogels performed under two consecutive cycles of such temperature change. Frequency sweep analysis of pure gelatin, Gel-Ada and Gel-Phe hydrogels performed at (c) $25^{\circ} \mathrm{C}$ and (d) $45^{\circ} \mathrm{C}$ (closed symbols represent the storage modulus $G^{\prime}$ and open symbols represent the loss modulus $G^{\prime \prime}$ ).

number, thereby further verifying the stability of the hydrogels during repeated cyclic temperature change (Supplementary Table S2). We believe that the slight increase in the storage modulus and loss modulus with increasing temperature cycles is due to the loss of water from the hydrogels. After immersing the samples in water for $30 \mathrm{~min}$ after the test, the storage and loss moduli of the hydrogels were restored to the initial levels (Supplementary Table S2).

The frequency sweep test further demonstrates the thermoplasticity of the Gel-Phe and Gel-Ada hydrogels (Figures $2 \mathrm{c}$ and d). At $25^{\circ} \mathrm{C}$, the storage modulus of both the Gel-Ada and Gel-Phe hydrogels is much higher than that of the pure gelatin hydrogels (Gel; Figure 2c). Moreover, the Gel-Phe and Gel-Ada hydrogels exhibit a more obvious frequency-dependent increase in the modulus than the pure gelatin hydrogels (Figure 2c). These results demonstrate that in addition to the tri-helix structure of gelatin, another form of physical crosslinking, hydrophobic aggregation, reinforces the hydrogels at room temperature. At $45^{\circ} \mathrm{C}$, the pure gelatin hydrogels turn into liquid solutions $\left(G^{\prime \prime}>G^{\prime}\right)$ due to the disassembly of the tri-helix structure (Figure $2 \mathrm{~d}$ ). The Gel-Phe and Gel-Ada hydrogels show slightly higher $G^{\prime}$ values than $G^{\prime \prime}$ values, and hydrophobic aggregation becomes the sole crosslinking mechanism of the hydrogels at high temperature (Figure 2d). In addition, the Gel-Ada and Gel-Phe hydrogels can be readily dissolved in DMSO but not in DI water at high temperature (Supplementary Figure S3), thereby further confirming the contribution of hydrophobic aggregations in stabilizing the hydrogels at high temperature.

The stretchability and toughness of supramolecular gelatin hydrogels

The Gel-Ada and Gel-Phe hydrogels also possess extraordinary mechanical properties. We performed tensile tests (elongation speed: $1 \mathrm{~mm} \mathrm{~s}^{-1}$ ) at room temperature to quantitatively measure the mechanical properties of the Gel-Ada and Gel-Phe hydrogels. Representative nominal stress vs $\lambda$ (stretch ratio: stretched length/original length) curves show that our Gel-Phe (prepared with $0.93 \mathrm{ml}$ phenyl isothiocyanate) and Gel-Ada (prepared with $1.5 \mathrm{~g} 1$-adamantyl isothiocyanate) hydrogels can be stretched to 42 and 34 times of their original length, respectively (Figures $3 a$ and $b$ ). Upon removal of the tensile force, the stretched hydrogels can recover their initial length immediately (Supplementary Videos 2 and 3). Furthermore, the rupture stress of these two hydrogels is over $1.5 \mathrm{MPa}$ (Figure 3c; Supplementary Videos 4 and 5). To the best of our knowledge, no previous studies have reported pure natural polymer-based hydrogels crosslinked only by physical interactions that can reach such high 
a
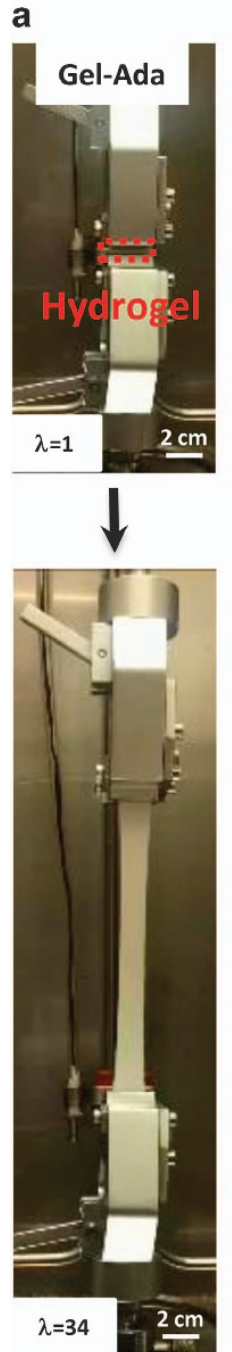

b
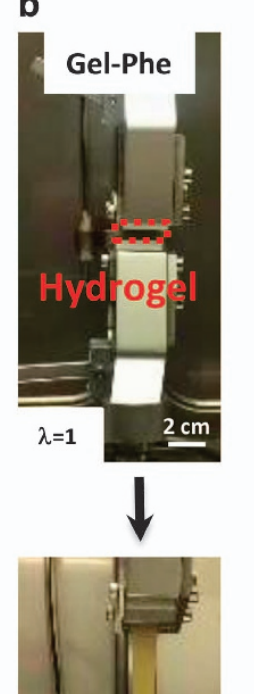

e 0.6

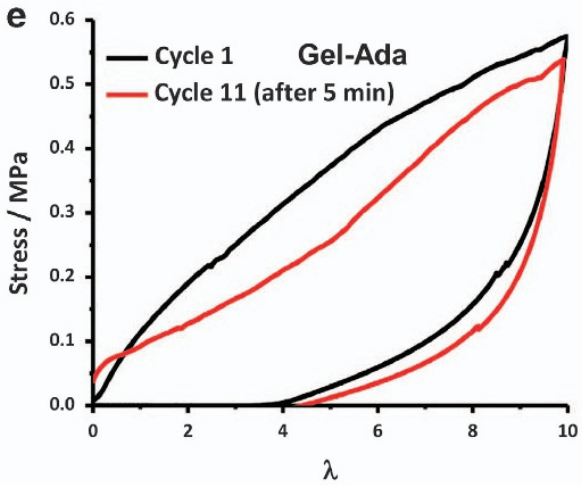

$91^{\text {st }}$ Test $\left(25^{\circ} \mathrm{C}\right)$

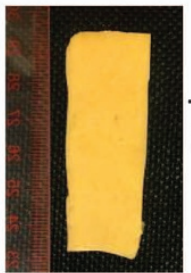

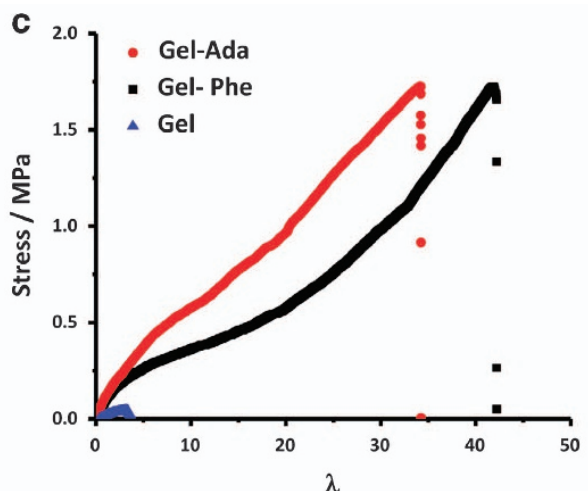

$\lambda$
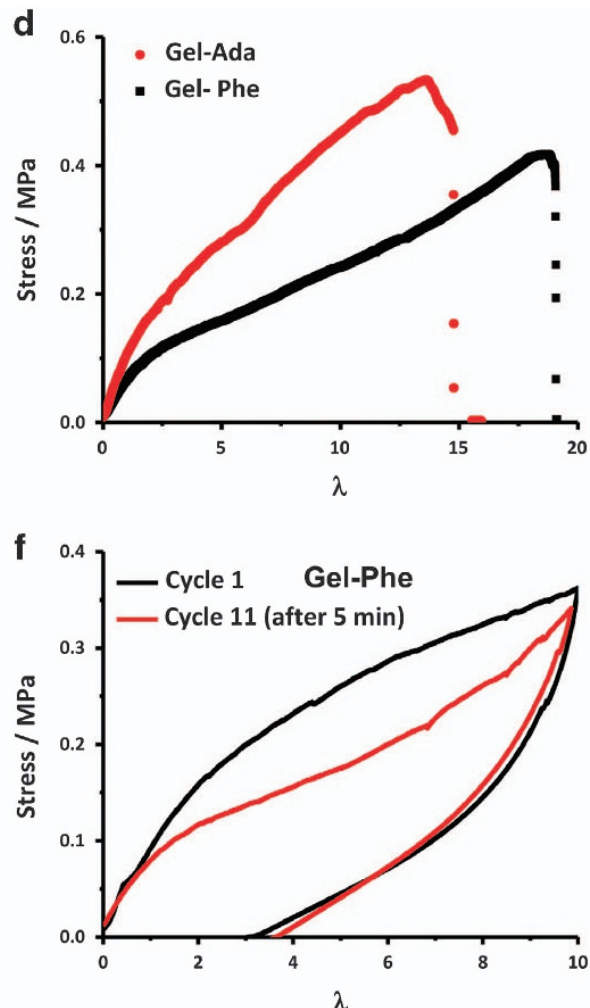

$2^{\text {st }}$ Test $\left(25^{\circ} \mathrm{C}\right)$
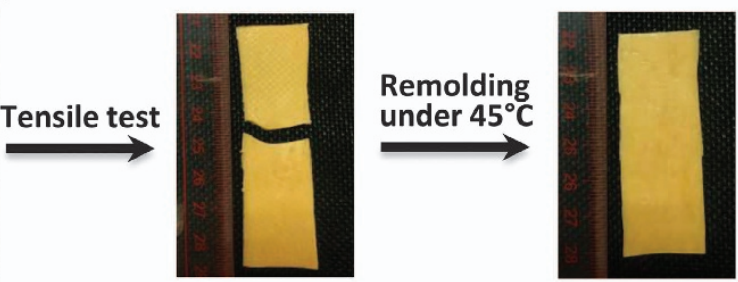

h

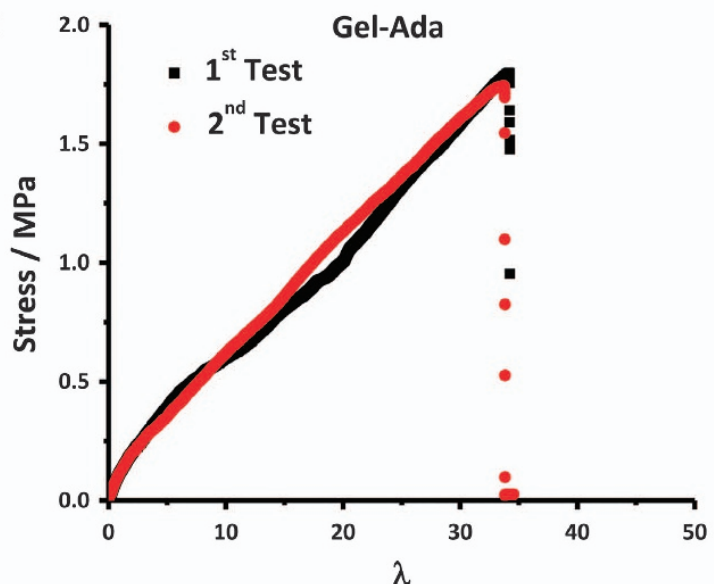

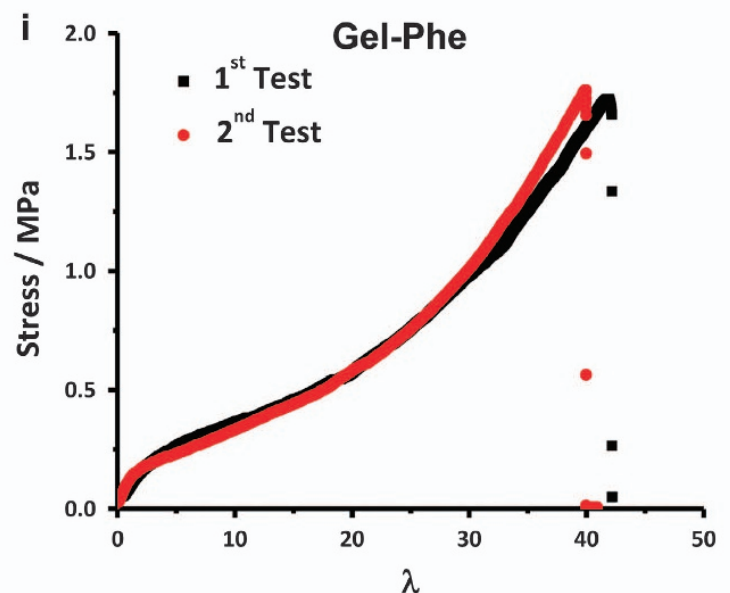

Figure 3 Tensile testing of the (a) Gel-Ada and (b) Gel-Phe hydrogels at room temperature. The stretch ratio $\lambda$ is defined by the length of stretched hydrogels divided by the original length of hydrogels. (c) Stress vs $\lambda$ curves of the pure gelatin, Gel-Ada and Gel-Phe hydrogels at room temperature. (d) Stress vs $\lambda$ curves of the notched Gel-Ada and Gel-Phe hydrogels at room temperature. After 10 continuous cycles of loading and unloading $(\lambda=10)$, the tested Gel-Adal Gel-Phe hydrogel samples were immersed in $25^{\circ} \mathrm{C}$ DI water for $5 \mathrm{~min}$ and then were tested again to evaluate the spontaneous recovery of the (e) Gel-Ada and (f) Gel-Phe hydrogels. The broken specimens of the (g) Gel-Phe and Gel-Ada hydrogels from the tensile test were remolded at high temperature, and the remolded and self-healed hydrogels exhibit almost identical tensile stiffness as that of the intact hydrogels $(\mathbf{h}, \mathbf{i})$. 
rupture stress, and strengthening with synthetic polymers can only improve the rupture modulus to a few hundreds of $\mathrm{kPa}$. The Gel-Ada and Gel-Phe hydrogels also show good tolerance to notches. The GelAda and Gel-Phe hydrogels with a $5 \mathrm{~mm}$ (20\% of the total width of the samples) notch in the middle of the samples can be stretched to 13 and 18 times their original length before rupture, respectively (Figure 3d; Supplementary Figure S10a and b; Supplementary Videos 6 and 7). Furthermore, based on the area under the stress vs $\lambda$ curves (below the strain at which the notch starts to propagate; Figure 3d; Supplementary Figure S10a), the fracture energies of the Gel-Ada and Gel-Phe hydrogels are estimated to be 2670 and $2460 \mathrm{~J} \mathrm{~m}^{-2}$, respectively, and these values are comparable to that of articular cartilage $\left(\sim 1000 \mathrm{~J} \mathrm{~m}^{-2}\right) .{ }^{39}$

Both the Gel-Ada and Gel-Phe hydrogels dissipate energy effectively, as evidenced by pronounced hysteresis during cyclic tensile testing. After the first cycle of loading and unloading, immediate application of the second cycle leads to reduced tensile stiffness in the Gel-Ada and Gel-Phe hydrogels, but this reduction in mechanical properties quickly diminishes with increasing cycle number, and the loading-unloading loops recorded after the sixth cycle closely overlap (Supplementary Figure S10c and d). Moreover, after 10 continuous loading-unloading cycles, a brief incubation of these hydrogels in DI water at room temperature for $5 \mathrm{~min}$ leads to over $90 \%$ recovery of the tensile properties of the hydrogel recorded in the first loading cycle (Figure $3 \mathrm{e}$ and f). We stretched the Gel-Ada and Gel-Phe hydrogels to 10 times their initial length to study the hydrogel stress relaxation under constant tensile strain for $10 \mathrm{~min}$. Supplementary Figure S10e shows that most of the stress relaxation occurred within the first $100 \mathrm{~s}$, and the percentage of stress relaxation in the Gel-Phe and Gel-Ada hydrogels after $10 \mathrm{~min}$ is $47 \%$ and $51 \%$, respectively. This finding demonstrates the substantial viscoelasticity of the Gel-Ada and GelPhe hydrogels. From the results of the tensile test, we speculate that the rigid tri-helix structure mainly contributes to the remarkable tensile resistance and elasticity, while the reversible hydrophobic aggregation affords substantial stretchability and viscosity of these two hydrogels. Meanwhile, the elastic tri-helix structure facilitates the recovery of hydrophobic aggregation during unloading.

The Gel-Ada and Gel-Phe hydrogels ruptured from the tensile tests can be completely healed because of their unique thermoplastic properties. Briefly, two fragments of ruptured Gel-Ada/Gel-Phe hydrogels were kneaded into a bulk hydrogel and then remolded in a $60 \times 20 \times 6 \mathrm{~mm}^{3}$ mold at high temperature $\left(45^{\circ} \mathrm{C}\right)$. After cooling to room temperature, the self-healed and remolded hydrogels have the same appearance as that of the original hydrogels (Figure 3g; Supplementary Figure S11). The entire process took $<5 \mathrm{~min}$. Furthermore, the remolded hydrogels exhibit almost identical tensile properties as the original hydrogels (Figure $3 \mathrm{~h}$ and i). These experiments further demonstrate that the Gel-Phe and Gel-Ada hydrogels can be recycled without the loss of mechanical properties. More importantly, because the Gel-Ada and Gel-Phe hydrogels are formed through the dialysis process in DI water, the as-prepared hydrogels should have reached swelling equilibrium. The equilibrium water content of the Gel-Phe and Gel-Ada hydrogels after 8 weeks of incubation in water are $75 \%$ and $70 \%$, respectively, which are identical to that of the as-prepared hydrogels (Supplementary Table S1). Therefore, immersing the Gel-Ada and Gel-Phe hydrogels in water does not affect their mechanical properties. In fact, we stored the GelAda and Gel-Phe hydrogels in water for days before conducting the mechanical tests described above.

\section{The adhesion and cytocompatibility of supramolecular gelatin hydrogels}

The adhesive properties of the Gel-Ada and Gel-Phe hydrogels are also remarkable. We used $1 \mathrm{ml}$ of the Gel-Ada (Figure 4a) or Gel-Phe (Supplementary Figure S12) hydrogels to adhere a hook to metal, glass, plastic and plastered wall surfaces at $45^{\circ} \mathrm{C}$. After cooling to room temperature, the hook can bear up to $2 \mathrm{~kg}$ of weight. Subsequent removal of the hydrogels at room temperature does not inflict any damage to the surface or leave residual adhered hydrogel. Furthermore, after removal from the surface, the hydrogels can adhere to the same surface or different surfaces after heating and support the weight again (we used the same piece of Gel-Phe/Gel-Ada hydrogel in Figure 4a; Supplementary Figure S11, respectively). We used the lap shear test to quantify the adhesive strength of these two hydrogels on metal, glass and tissue (porcine skin) surfaces. As shown in Figure 4b, the adhesive stress of the Gel-Ada hydrogels reaches almost $300 \mathrm{kPa}$. Although the adhesive stress on tissue is lower than that on the metal and glass surfaces, the value is still much higher than that of the widely

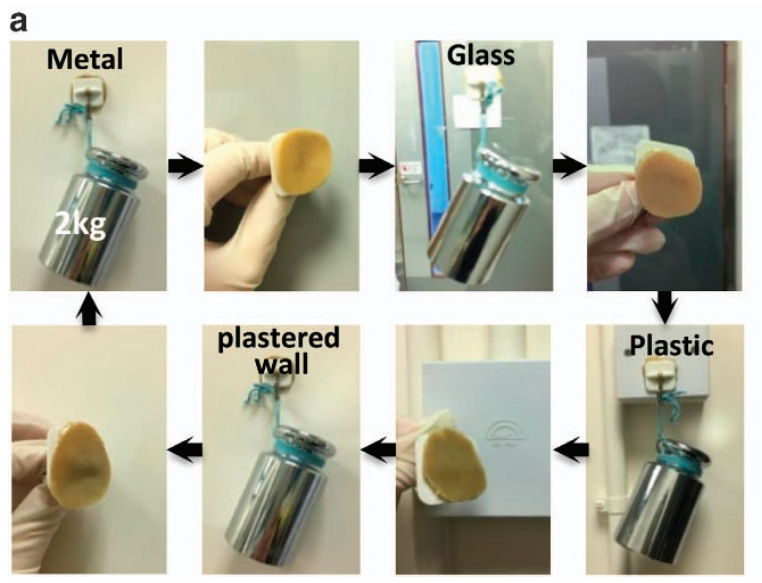

b

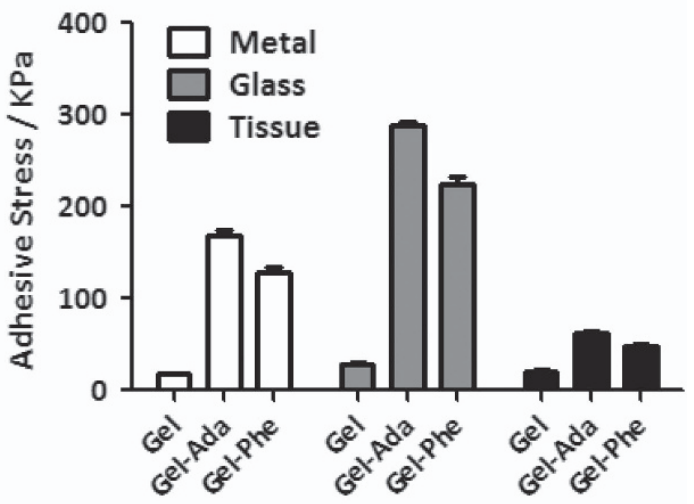

Figure 4 (a) Adhesive properties of the Gel-Phe hydrogels: the Gel-Phe hydrogels can be used to glue a hook to different surfaces (metal, glass, plastic and plastered wall) at high temperature. After the hydrogel was cooled to room temperature, the hook can support 2 kg of weight. Furthermore, the hydrogel can be removed from the surface without damaging the surface and can be reused as glue after heating. The same piece of Gel-Phe hydrogel was consecutively used to adhere to different surfaces. (b) The adhesive stress of the pure gelatin, Gel-Ada and Gel-Phe hydrogels with different surfaces (metal, glass and tissue) was quantified by the lap-shear test at room temperature. 
used commercial bioglue, fibrin glue $(7 \mathrm{kPa}) .{ }^{40}$ Moreover, the adhesive stress of the pure gelatin hydrogel is $\sim 20 \mathrm{kPa}$ at room temperature, which is much lower than that of our Gel-Ada and Gel-Phe hydrogels (Figure 4b). We speculate that the excellent adhesion of the Gel-Ada and Gel-Phe hydrogels to different materials can be attributed to two factors: (1) hydrogen bonding between the gelatin polymers and the material surface leads to the formation of an adhesive interface; and (2) the outstanding mechanical properties of the hydrogels based on hydrophobic aggregation and the gelatin tri-helix structure lead to the high cohesion strength of the hydrogels, thereby significantly improving the overall adhesion. The second factor appears to be the dominant factor, as evidenced by the significantly higher adhesion of the Gel-Ada and Gel-Phe hydrogels compared with the pure gelatin hydrogels. To assess the biocompatibility of the Gel-Ada and Gel-Phe hydrogels, we incubated the Gel-Ada and Gel-Phe hydrogels $(0.5 \mathrm{ml}$ volume) in the culture media of hMSCs (passage 4). After 5 and 10 days of culture, cell live/dead staining shows that the Gel-Ada and Gel-Phe hydrogels have little effect on the viability of hMSCs compared to the control group without hydrogels (Supplementary Figure S13).

\section{CONCLUSION}

Collectively, the present work demonstrates a simple and effective onepot approach based on solvent exchange to prepare natural polymeric supramolecular hydrogels with dual physical crosslinking mechanisms, namely, self-assembly of the gelatin tri-helix structure and aggregation of the free and grafted hydrophobic motifs. Because of the synergistic action of these two physical crosslinking mechanisms, the resulting supramolecular gelatin hydrogels exhibit unique thermoplastic behavior, excellent tensile properties and toughness. These supramolecular gelatin hydrogels also show outstanding adhesion to various surfaces as well as biocompatibility. It is remarkable that these excellent mechanical properties can be achieved in supramolecular hydrogels under the swollen equilibrium state with over $70 \%$ water content. These desirable properties make our supramolecular gelatin hydrogels promising materials for potential biomedical applications. Our ongoing work aims to further improve the mechanical functions of these hydrogels at physiological temperature.

\section{CONFLICT OF INTEREST}

The authors declare no conflict of interest.

\section{ACKNOWLEDGEMENTS}

Project 31570979 and Project 31370949 are supported by the National Natural Science Foundation of China. The work described in this paper is supported by a General Research Fund grant from the Research Grants Council of Hong Kong (Project No. 14220716). This work is supported by the Health and Medical Research Fund, Food and Health Bureau, Government of the Hong Kong Special Administrative Region (reference no.: 03140056). This research is supported by the Chow Yuk Ho Technology Centre for Innovative Medicine, The Chinese University of Hong Kong. This work is also supported by the National Key Technology R\&D Program of China (2016YFC1102305). This research is also supported by project BME-p3-15 of the Shun Hing Institute of Advanced Engineering, The Chinese University of Hong Kong.

\section{PUBLISHER'S NOTE}

Springer Nature remains neutral with regard to jurisdictional claims in published maps and institutional affiliations.
1 Chaudhuri, O., Gu, L., Klumpers, D., Darnell, M., Bencherif, S. A., Weaver, J. C., Huebsch, N., Lee, H., Loppens, E., Duda, G. N. \& Mooney, D. J. Hydrogels with tunable stress relaxation regulate stem cell fate and activity. Nat. Mater. 15, 326-334 (2016).

2 Han, L., Lu, X., Liu, K., Wang, K., Fang, L., Weng, L. T., Zhang, H., Tang, Y., Ren, F., Zhao, C., Sun, G., Liang, R. \& Li, Z. Mussel-inspired adhesive and tough hydrogel based on nanoclay confined dopamine polymerization. ACS Nano 11, 2561-2574 (2017).

3 Tian, K., Bae, J., Bakarich, S. E., Yang, C., Gately, R. D., Spinks, G. M., In Het Panhuis, M., Suo, Z. \& Vlassak, J. J. 3D printing of transparent and conductive heterogeneous hydrogel-elastomer systems. Adv. Mater. 29, 1604827 (2017).

4 Tay, C. Y., Wu, Y. L., Cai, P., Tan, N. S., Venkatraman, S. S., Chen, X. \& Tan, L. P. Bioinspired micropatterned hydrogel to direct and deconstruct hierarchical processing of geometry-force signals by human mesenchymal stem cells during smooth muscle cell differentiation. NPG Asia Mater. 7, e199 (2015).

$5 \mathrm{Wu}, \mathrm{Z}$. L. \& Gong, J. P. Hydrogels with self-assembling ordered structures and their functions. NPG Asia Mater. 3, 57-64 (2011).

6 Cheng, H., Hu, C., Zhao, Y. \& Qu, L. Graphene fiber: a new material platform for unique applications. NPG Asia Mater. 6, e113 (2014).

7 Takashima, Y., Sawa, Y., Iwaso, K., Nakahata, M., Yamaguchi, H. \& Harada, A. Supramolecular materials cross-linked by host-guest inclusion complexes: the effect of side chain molecules on mechanical properties. Macromolecules 50, 3254-3261 (2017)

8 Sun, J. Y., Zhao, X., Illeperuma, W. R., Chaudhuri, O., Oh, K. H., Mooney, D. J., Vlassak, J. J. \& Suo, Z. Highly stretchable and tough hydrogels. Nature 489, 133-136 (2012)

9 Barrow, S. J., Kasera, S., Rowland, M. J., del Barrio, J. \& Scherman, O. A. Cucurbiturilbased molecular recognition. Chem. Rev. 115, 12320-12406 (2015).

10 Kakuta, T., Takashima, Y. \& Harada, A. Highly elastic supramolecular hydrogels using host-guest Inclusion complexes with cyclodextrins. Macromolecules 46, 4575-4579 (2013).

11 Xia, L.-W., Xie, R., Ju, X.-J., Wang, W., Chen, Q. \& Chu, L.-Y. Nano-structured smart hydrogels with rapid response and high elasticity. Nat. Commun. 4, 2226 (2013)

$12 \mathrm{Li}$, J. Self-assembled supramolecular hydrogels based on polymer-cyclodextrin inclusion complexes for drug delivery. NPG Asia Mater. 2, 112-118 (2010).

13 Voorhaar, L., Diaz, M. M., Leroux, F., Rogers, S., Abakumov, A. M., Tendeloo, G. V., Assche, G. V., Mele, B. V. \& Hoogenboom, R. Supramolecular thermoplastics and thermoplastic elastomer materials with self-healing ability based on oligomeric charged triblock copolymers. NPG Asia Mater. 9, e385 (2017).

14 Wang, Q., Mynar, J. L., Yoshida, M., Lee, E., Lee, M., Okuro, K., Kinbara, K. \& Aida, T. High-water-content mouldable hydrogels by mixing clay and a dendritic molecular binder. Nature 463, 339-343 (2010).

15 Ishii, S., Kokubo, H., Hashimoto, K., Imaizumi, S. \& Watanabe, M. Tetra-PEG network containing ionic liquid synthesized via Michael addition reaction and its application to polymer actuator. Macromolecules 50, 2906-2915 (2017).

16 Zhang, H. J., Sun, T. L., Zhang, A. K., Ikura, Y., Nakajima, T., Nonoyama, T., Kurokawa, T., Ito, O., Ishitobi, H. \& Gong, J. P. Tough physical double-network hydrogels based on amphiphilictriblock copolymers. Adv. Mater. 28, 4884-4890 (2016).

17 Xu, B., Zheng, P., Gao, F., Wang, W., Zhang, H., Zhang, X., Feng, X. \& Liu, W. A mineralized high strength and tough hydrogel for skull bone regeneration. Adv. Funct. Mater. 27, 1604327 (2016).

18 Shi, Z., Gao, H., Feng, J., Ding, B., Cao, X., Kuga, S., Wang, Y., Zhang, L. \& Cai, J. In situ synthesis of robust conductive cellulose/polypyrrole composite aerogels and their potential application in nerve regeneration. Angew. Chem. Int. Ed. 53, 5380-5384 (2014).

19 Han, L., Yan, L., Wang, K., Fang, L., Zhang, H., Tang, Y., Ding, Y., Weng, L. T., Xu, J., Weng, J., Liu, Y., Ren, F. \& Lu, X. Tough, self-healable and tissue-adhesive hydrogel with tunable multifunctionality. NPG Asia Mater. 9, e372 (2017).

20 Liu, D., Cheng, E. \& Yang, Z. DNA-based switchable devices and materials. NPG Asia Mater. 3, 109-114 (2011).

21 Hsieh, F.-Y., Tao, L., Wei, Y. \& Hsu, S.-h. A novel biodegradable self-healing hydrogel to induce blood capillary formation. NPG Asia Mater. 9, e363 (2017).

22 Solanki, P. R., Kaushik, A., Agrawal, V. V. \& Malhotra, B. D. Nanostructured metal oxide-based biosensors. NPG Asia Mater. 3, 17-24 (2011).

23 Sun, T. L., Kurokawa, T., Kuroda, S., Ihsan, A. B., Akasaki, T., Sato, K., Haque, M. A., Nakajima, T. \& Gong, J. P. Physical hydrogels composed of polyampholytes demonstrate high toughness and viscoelasticity. Nat. Mater. 12, 932-937 (2013).

24 Kakuta, T., Takashima, Y., Nakahata, M., Otsubo, M., Yamaguchi, H. \& Harada, A. Preorganized Hydrogel: self-healing properties of supramolecular hydrogels formed by polymerization of host-guest-monomers that contain cyclodextrins and hydrophobic guest groups. Adv. Mater. 25, 2849-2853 (2013).

25 Dai, X., Zhang, Y., Gao, L., Wang, W., Cui, Y. \& Liu, W. Mechanically strong, highly stable, thermoplastic, and self-healable supramolecular polymer hydrogel. Adv. Mater. 27, 3566-3571 (2015).

26 Wei, K., Zhu, M., Sun, Y., Xu, J., Feng, Q., Lin, S., Wu, T., Xu, J., Tian, F., Xia, J., Li, G. \& Bian, L. Robust biopolymeric supramolecular "host-guest macromer" hydrogels reinforced by in situ formed multivalent nanoclusters for cartilage regeneration. Macromolecules 49, 866-875 (2016).

27 Feng, Q., Wei, K., Lin, S., Xu, Z., Sun, Y., Shi, P., Li, G. \& Bian, L. Mechanically resilient, injectable, and bioadhesive supramolecular gelatin hydrogels crosslinked by 
weak host-guest interactions assist cell infiltration and in situ tissue regeneration. Biomaterials 101, 217-228 (2016).

28 Kamata, H., Akagi, Y., Kayasuga-Kariya, Y., Chung U.-i. \& Sakai, T. "Nonswellable" hydrogel without mechanical hysteresis. Science 343, 873-875 (2014).

29 Becher, T. B. \& Ornelas, C. Nonswellable injectable hydrogels self-assembled through non-covalent interactions. ChemistrySelect 2, 3009-3013 (2017).

30 Nakagawa, S., Li, X., Kamata, H., Sakai, T., Gilbert, E. P. \& Shibayama, M. Microscopic structure of the "nonswellable" thermoresponsive amphiphilic conetwork. Macromolecules 50, 3388-3395 (2017).

31 Guvendiren, M. \& Shull, K. R. Self-assembly of acrylic triblock hydrogels by vapor-phase solvent exchange. Soft Matter 3, 619-626 (2007).

32 Bai, S., Nguyen, T. L., Mulvaney, P. \& Wang, D. Using hydrogels to accommodate hydrophobic nanoparticles in aqueous media via solvent exchange. Adv. Mater. 22, 3247-3250 (2010).

33 Zheng, J., Jung, S., Schmidt, P. W., Lodge, T. P. \& Reineke, T. M. 2-Hydroxyethylcellulose and amphiphilic block polymer conjugates form mechanically tunable and nonswellable hydrogels. ACS Macro Lett. 6, 145-149 (2017).

34 Bigi, A., Panzavolta, S. \& Rubini, K. Relationship between triple-helix content and mechanical properties of gelatin films. Biomaterials 25, 5675-5680 (2004).

35 Guo, L., Colby, R. H., Lusignan, C. P. \& Whitesides, T. H. Kinetics of triple helix formation in semidilute gelatin solutions. Macromolecules 36 9999-10008 (2003).

36 Sahoo, S. K., Mallick, B., Nath, I. \& Mukharjee, R. N. Effect of mev electron beam irradiation on fracture healing of tibia bone of rabbit. Int. J. Biol. Med. Res. 5 4569-4577 (2014).

37 Zhao, X., Lang, Q., Yildirimer, L., Lin, Z. Y., Cui, W., Annabi, N., Ng, K. W., Dokmeci, M. R., Ghaemmaghami, A. M. \& Khademhosseini, A. Photocrosslinkable gelatin hydrogel for epidermal tissue engineering. Adv. Healthc. Mater. 5, 108-118 (2016).

38 Neffe, A. T., Pierce, B. F., Tronci, G., Ma, N., Pittermann, E., Gebauer, T., Frank, 0. Schossig, M., Xu, X., Willie, B. M., Forner, M., Ellinghaus, A., Lienau, J., Duda, G. N. \& Lendlein, A. One step creation of multifunctional 3D architectured hydrogels inducing bone regeneration. Adv. Mater. 27, 1738-1744 (2015).

39 Simha, N., Carlson, C. \& Lewis, J. Evaluation of fracture toughness of cartilage by micropenetration. J. Mater. Sci. Mater. Med. 15, 631-639 (2004).

40 Wang, R., Ki, J., Chen, W., Xu, T., Yun, S., Xu, Z., Xu, Z., Sato, T., Chi, B. \& Xu, H. A biomimetic mussel-inspired $\varepsilon$-poly-l-lysine hydrogel with robust tissue-anchor and anti-infection capacity. Adv. Funct. Mater. 27, 1604894 (2017).

\section{(c) (i)}

This work is licensed under a Creative Commons Attribution 4.0 International License. The images or other third party material in this article are included in the article's Creative Commons license, unless indicated otherwise in the credit line; if the material is not included under the Creative Commons license, users will need to obtain permission from the license holder to reproduce the material. To view a copy of this license, visit http:// creativecommons.org/licenses/by/4.0/

(C) The Author(s) 2018

Supplementary Information accompanies the paper on the NPG Asia Materials website (http://www.nature.com/am) 